\title{
Viewpoints
}

\section{Implementing policies and programmes to reduce the impact of globalisation on oral health in Pacific Island Countries and Territories}

\author{
Nafanua Pili', Vili Nosa² ${ }^{1}$, Leroy Tatui ${ }^{3}$ \\ ${ }^{1}$ Ministry of Health New Zealand, 2 Pacific Health Section, School of Population Health, Faculty of Medical \& Health Sciences, University of Auckland, 3 \\ Niue Foou Hospital \\ Keywords: Pacific Islands Countries and Territories, Pacific oral health, Pacific communities \\ https://doi.org/10.52872/001c.29655
}

Journal of Global Health Economics and Policy

Vol. 1, 2021

\begin{abstract}
Very little research focuses explicitly on oral health in Pacific Islands countries and territories (PICT). However, oral health will continue to be a concern if policies, programmes, and strategies are not implemented to reduce poor oral health and inequitable outcomes that will affect many Pacific communities. A review of available studies written between 1969 and 2018 shows that dental caries and periodontal diseases were identified as the most significant oral health issue in PICT. The globalisation of imported food products that are high in sugary content has further exacerbated the increase in DMFT (decayed, missing or filled teeth). With the introduction of tobacco, there have also been many oral health issues with oral cancer, periodontal disease, and periodontitis. There is a need for a sugar tax policy, food policy changes, tobacco policy and the removal tax of oral health products in PICT. In addition, most oral health services are provided in the hospitals and often work within their own departments whereas they might be better working closely with other departments such as the public health team or the education sector so, therefore, there needs to be a coordinated collaboration approach with other services that can promote good oral health policies and health promotion activities.
\end{abstract}

\section{INTRODUCTION}

According to the FDI World Dental Foundation, oral health is multi-faceted and includes the ability to speak, smile, smell, taste, touch, chew, swallow and convey a range of emotions through facial expressions with confidence and without pain, discomfort and disease of the craniofacial complex. ${ }^{1}$ Much of the literature highlights the increasing burden of oral health as a growing public health issue with oral diseases spreading across all regions of the globe. ${ }^{2,3}$ In particular, the prevalence of dental caries and periodontal diseases bring the greatest concern. This is especially true among those living in Pacific Island Countries and Territories (PICTs). The literature around oral health in PICTs points to globalisation as the primary catalyst for the increasing prevalence of oral diseases across PICTs. ${ }^{4}$ The aim of this paper is a narrative review to explore policies and programmes to reduce the impact of globalisation on oral health in Pacific Island Countries and Territories, focused on the analysis of the previously published papers.

\section{MAIN FINDINGS}

Dental caries and periodontal diseases have been identified as the most significant oral health issues faced by PICTs. ${ }^{5-7}$ The prevalence of dental caries is generally measured through the DMFT (decayed, missing or filled teeth) index, which helps quantify the state of oral health. ${ }^{8} \mathrm{~A}$ common theme seen throughout these countries is the high and increasing rate of tooth decay among children. When compared with children in the United States of America (US), the Federated States of Micronesia (FSM), ${ }^{9}$ the Republic of Marshall Islands (RMI), ${ }^{10}$ Guam and Palau show consistently higher rates of mean DMFT. Although there has been a general decline in dental caries in US children over time, the rate of dental caries among these Micronesian nations appears to be increasing. ${ }^{9-11}$ For example, an Oral Health Survey of children in the US saw a mean decrease of DMFT rates in 12-year-olds by 36 percent from 4.18 in $1979-89$ to 2.66 in $1986-87 .{ }^{12}$

In contrast, FSM saw a 21 percent increase of DMFT rates between 1976-77 and 1986-87 from 4.88 to 5.91 among 12-year-olds in FSM. ${ }^{9}$ Additionally, research published by Greer et al., ${ }^{11}$ found that the age-weighted mean for children (aged 5-9 years old) in Guam and Palau were 3.1 and 2.9 times higher than the US national mean. Similar trends can be found across islands in the Melanesian region, such as New Caledonia, ${ }^{12}$ and Fiji. ${ }^{4}$ For example, although the prevalence rates for dental caries have improved among older children, a study found that approximately 60 percent of New Caledonian 6-year-olds had at least one deciduous or permanent tooth with untreated caries compared to only 33 percent of French 6-year-olds. ${ }^{12}$ In Fiji, indigenous chil- 
dren who live in rural areas hold the highest DMFT rates, increasing from 62 percent to 82 percent within a 15 -year period. 4

Polynesian countries such as the Cook Islands, Tokelau, Samoa, American Samoa, Tonga, and Tuvalu are no exception to the caries epidemic. ${ }^{4,13}$ Across these six nations, there has been an increase in dental caries, with the younger generation being most at risk. ${ }^{4}$ As in the aforementioned Melanesian territories, these Polynesian nations show signs of increased caries levels in children. Additionally, the Cook Islands has identified an increase in the prevalence of dental caries with age. ${ }^{4}$ In the Cook Islands, a study conducted in Rarotonga highlighted that only 12 percent of 5-year-olds had experienced tooth decay compared to approximately 100 percent of 15 -year-olds. ${ }^{4} \mathrm{Sim}$ ilarly, in the island of Mangaia, 0 percent of 5 -year-olds had experienced dental caries compared to 89 percent of 15 -year-olds; despite being most prevalent among children, untreated dental caries can be dangerous and lead to further complications during adulthood. For instance, dental caries is a major source of missing teeth among adults in the Cook Islands, Tokelau, and Tonga. ${ }^{4}$

Compared to dental caries, there is less information surrounding periodontal diseases in the Pacific despite being around longer. ${ }^{5}$ Information on the prevalence of periodontal disease is limited, with most dental services in PICTs focused on treating dental caries. ${ }^{5}$ Most of the literature is from Polynesian countries such as the Cook Islands, Tokelau, Tonga, and Samoa regarding periodontal diseases. It was difficult to source any information from their Micronesian and Melanesian neighbours, with Fiji being the exception; the available research shows that in PICTs, those most affected by periodontal diseases are adults. ${ }^{5,6,14}$ In the Cook Islands, it was found that 92 percent of those over the age of 40 -years-old were affected with gum disease. ${ }^{5}$ Similarly, in Tokelau, over 90 percent of adults aged over 55 -years-old showed gingivitis/calculus/periodontitis complex. ${ }^{5}$ The literature highlights that periodontal disease escalates rapidly with age and should be avoided through well-established oral hygiene habits. ${ }^{5}$

\section{THE GLOBALISATION OF FOOD POLICIES}

In previous decades, dental caries was very uncommon among indigenous populations in the Pacific Islands Countries and Territories. Pacific people were well known for their picture-perfect teeth and pristine periodontal health. ${ }^{4}$ The expansion of European trading and migration across the region resulted in an increased sugar and refined carbohydrates intake within PICTs. As a result, there was a major shift in traditional diets, particularly in food preparation and lifestyle choices. ${ }^{4,15}$ Initially, this was an issue only seen in more urban areas of the islands; however, with the increase of trading and cash economies, the availability of highly processed sugary foods and ingredients further spread to rural areas. ${ }^{4,16}$ Change in lifestyle and dietary habits in PICTs are the most associated with the increase of dental caries and are closely related to other chronic diseases such as diabetes, cardiovascular disease and cancer. ${ }^{15,16}$ The burden linked to these chronic diseases is felt significantly by PICT populations and often overshadow is- sues concerning oral health. There is little in the literature about social class in the Pacific, and whether there is an association between socioeconomic status and diet, a pattern seen in Western countries. ${ }^{17}$ There is also the need to investigate how food policies can reduce poor oral health.

\section{INFLUENCE OF TOBACCO POLICIES}

The introduction of tobacco through globalisation has also contributed significantly to the rise of oral health issues across the Pacific region. ${ }^{5}$ Although tobacco use has decreased in most developed countries, there is an increasing trend in low-income and middle-income countries. ${ }^{2}$ In particular, smoking has been identified as a major risk factor for oral cancer, periodontal disease, and periodontitis. Similarly to changing diets, the increase in smoking across PICTs also negatively affects the population's general health and increases their susceptibility to other diseases such as lung cancer. ${ }^{2,5,6}$ Consistent brushing and self-care have been identified as practical ways of reducing periodontal disease and oral health issues. However, these methods could be more effective if implemented in conjunction with broader policy changes to prevent the increase of oral health risks such as tobacco. ${ }^{6}$

\section{CONCLUSIONS}

This review provides a general picture of the state of oral health across PICTs. According to Cutress, ${ }^{5}$ historically, PICTs were known for the immaculate condition of their teeth. However, the effects of globalisation have seen an increase in foods high in sugar and tobacco consumption. Consequently, those in the Pacific have experienced a rapid rise in dental caries among children and periodontal disease in adults. ${ }^{4}$ It is essential to address these issues as failure to take timely action may eventually lead to hospitalisation and further complications later in life. This not only places unnecessary costs on the health system but increases the number of preventable health issues. ${ }^{3,16}$ It is unlikely and unrealistic to expect PICTs to revert to their traditional diets. This offers an excellent opportunity to implement oral health policies, preventative interventions that promote positive dietary behaviours and better lifestyle choices from a political and structural level. ${ }^{18}$ A concept emphasised throughout the literature is the need to focus more on oral health policies and health promotion that enable PICTs to partake in better oral health practices. ${ }^{6}$ Solutions found in the literature include a demand for governments to take responsibility by ensuring that healthy public policies are implemented. Some recommendations that have been made are the imposition of a sugar tax, removing tax off oral health products (i.e., fluoridated toothpaste), and enforcing formal oral health promotion strategies within general health promotion. This needs to be actively supported and promoted by governments in order to reach full effectiveness. ${ }^{6,18}$ This could be highly beneficial for the overall health outcomes of PICTs as the risk factors for oral health issues and non-communicable diseases are similar. ${ }^{2,10}$

Working in a multi- or inter-sectoral manner is another standard recommendation made throughout the literature. 
This includes involving other government agencies and stakeholders that work outside the traditional oral health team. 2,6,18 Most oral health services in PICTs operate in hospitals, creating an ideal environment for cross-sectoral collaboration; however, oral health is often viewed in isolation from other departments within the hospitals. The isolation and lack of resources and workforce make it difficult for oral health services in PICTs to provide preventive or restorative dental care and address the risk factors that stem from globalisation. ${ }^{2,3}$ There is a significant opportunity to reorient oral health policies and services by encouraging collaboration and implementing the common risk factor approach. Creating collaborative networks and standard policy changes may be simplistic and face economic, political, and structural setbacks. However, it has the extreme potential to help overcome issues of scarce resources and address the complexity of oral health. ${ }^{18}$

Some of the literature is outdated, which can be problematic as the information synthesised from the research may not accurately reflect the state of oral health in PICTs today. In addition, the availability of data within the literature is quite limited, with a lack of consistency across countries in terms of measuring and monitoring oral health information. This makes it difficult to form clear comparisons between PICTs and to draw any meaningful conclusions. However, despite the lack of current and consistent data, the information received from the literature is the best source of publicly available information and paints a general picture of oral health within the Pacific Islands Countries and Territories.

Dental caries and periodontal diseases are the leading oral health issues identified in the literature for Pacific Islands Countries and Territories. The effects of globalisation on food policies and the introduction of tobacco are critical contributors to the rise in oral health diseases within this region. Research from the literature urges the need to take a multi-level approach to encourage better oral hygiene and implement public policy programmes that address these more constitutional risk factors. These policy changes will help inform oral health policies, oral health service planning and oral health management across the Pacific Islands Countries and Territories.

\section{ACKNOWLEDGEMENTS}

The authors want to thank the University of Auckland Summer Research Scholarship funders.

\section{ETHICAL APPROVAL}

No ethical approval was required for this paper.

\section{FUNDING}

Funding was received for this study from the University of Auckland Summer Research Scholarship.

\section{AVAILABILITY OF DATA}

Data used in this study is available upon reasonable request to the authors.

\section{AUTHOR'S CONTRIBUTION}

All three authors contributed to the design, acquisition of data, analysis and interpretation of the data, drafting of the manuscript and gave approval of the final version and have agreed to be accountable for all aspects of the work.

\section{COMPETING INTERESTS}

The authors completed the ICMJE Unified Competing Interest form at (available upon request from the corresponding author) and declare no conflicts of interest.

\section{AUTHORS INFORMATION}

Nafanua Pili is a Policy analyst at the Ministry of Health New Zealand. Nafanua has an interest in Pacific health equitable issues.

Associate Professor Vili Nosa is the Head of Pacific Section, and an Associate Professor in Pacific Health at the University of Auckland. He has an interest in Pacific health issues in the Pacific region and New Zealand.

Leroy Tatui is the Principal Dental officer at Niue Foou Hospital, Niue Islands. He has an interest in improving oral health services in Niue and for the Pacific region.

\section{CORRESPONDENCE}

Associate Professor Vili Nosa, Pacific Health Section, School of Population Health, Faculty of Medical \& Health Sciences, The University of Auckland, Auckland, New Zealand v.nosa@auckland.ac.nz

Submitted: October 05, 2021 CET, Accepted: October 25, 2021 CET 


\section{REFERENCES}

1. Glick M, Williams DM, Kleinman DV, Vujicic M, Watt RG, Weyant RJ. A new definition for oral health developed by the FDI World Dental Federation opens the door to a universal definition of oral health. Journal of Public Health Dentistry. 2017;77(1):3-5. 으 i:10.1111/jphd.12213

2. Petersen PE. The World Oral Health Report 2003: continuous improvement of oral health in the 21 st century--the approach of the WHO Global Oral Health Programme. Community Dent Oral Epidemiol. 2003;31(Suppl 1):3-23. doi:10.1046/j..2003.com122.x

3. Haag DG, Peres KG, Balasubramanian M, Brennan DS. Oral Conditions and Health-Related Quality of Life: A Systematic Review. J Dent Res. 2017;96(8):864-874. doi:10.1177/0022034517709737

4. Cutress TW. Dental caries in South Pacific populations: a review. Pacific health dialog. 2003;10(1):62-67.

5. Cutress TW. Periodontal health in South Pacific populations: a review. Pac Health Dialog. 2003;10(1):68-75.

6. Tuisuva J, Pushpaangaeli B, Morse Z. Pacific oral health summit--"A call for action", 2001. Pac Health Dialog. 2003;10(1):111-113.

7. Finau SA. Oral health and personnel needs in the Pacific. Aust Dent J. 1996;41(1):53-58. doi:10.1111/j.1 834-7819.1996.tb05656.x

8. Pulu AI. Niue public dental health services: an evaluation 1965-1983. https://ses.library.usyd.edu.au/ bitstream/handle/2123/4213/0431.pdf?sequence $=4$.

9. Louie R. Developing vs Importing Oral Health Resources: The PHS Experience in the Pacific. J Public Health Dent. 1994;54(4):238-241. doi:10.1111/j.175 2-7325.1994.tb01223.x
10. Tut OK, Langidrik JR, Milgrom PM. Dental manpower development in the Pacific: case study in the Republic of the Marshall Islands. Pac Health Dialog. 2007;14(1):245-250.

11. Greer MH, Larson K, Sison S. Comparative analysis of oral health indicators among young children in Hawai'i, the Republic of Palau and Territory of Guam, 1999-2000. Pac Health Dialog. 2003;10(1):6-11.

12. Pichot H, Hennequin M, Rouchon B, Pereira B, Tubert-Jeannin S. Dental status of new caledonian children: is there a need for a new oral health promotion programme? PLOS ONE. 2014;9(11):e112452. doi:10.1371/journal.pone.01124 $\underline{52}$

13. Speake JD, Malaki T. Oral health in Tuvalu. Commun Dent Oral Epidemiol. 1982;10(4):173-177. do i:10.1111/j.1600-0528.1982.tb00374.x

14. Malolo ML. Changing Patterns of Oral Disease Prevalence In Tonga. https://ses.library.usyd.edu.au/h andle/2123/4407.

15. Doherty MA, Blinkhorn AS, Vane ES. Oral health in the Pacific Islands. Int Dent J. 2010;60(2):122-128.

16. Aldwell K, Caillaud C, Galy O, Frayon S, AllmanFarinelli M. Tackling the Consumption of High Sugar Products among Children and Adolescents in the Pacific Islands: Implications for Future Research. Healthcare (Basel). 2018;6(3):81. doi:10.3390/healthca $\underline{\text { re6030081 }}$

17. Otero G, Pechlaner G, Liberman G, Gürcan E. The neoliberal diet and inequality in the United States. Soc Sci Med. 2015;142:47-55. doi:10.1016/j.socscime d.2015.08.005

18. Tatui L, Mccool J, Nosa V. Rethinking and establishing a dental collaboration in the Pacific region. Pac Health Dialog. 2018;21(2):108-110. doi:1 $\underline{0.26635 / p h d .2018 .921}$ 